\title{
ESTIMACIÓN DEL COSTO DEL PRIMER RALEO DE AlgarRobo BLANCO (PROSOPIS ALBA): METODOLOGÍA APLICADA AL CASO DE UNA plantación en Herrera, Santiago del Estero, Argentina
}

\author{
Marta Coronel de Renolfi1 ${ }^{1}$ Adriana T. Gómez ${ }^{2}$
}

${ }^{1}$ Profesor asociado e investigador, Facultad de Ciencias Forestales Universidad Nacional de Santiago del Estero, Argentina Correo electrónico: mrenolf@unse.edu.ar 2 Investigador, Instituto Nacional de Tecnología Agropecuaria (INTA), Santiago del Estero, Argentina

Recibido: 10 de mayo del 2014. Aprobado: 15 de agosto del 2014.

Cómo citar este artículo: M. Coronel de Renolfi y A. T. Gómez. "Estimación del costo del primer raleo de algarrobo blanco (Prosopis alba): metodología aplicada al caso de una plantación en Herrera, Santiago del Estero, Argentina". Ingeniería Solidaria, vol. 10, n. 17, pp. 19-27, en.-dic., 2014. doi: http://dx.doi.org/10.16925/ in.v9i17.790

Resumen. Se analizó la operación del primer raleo forestal en una plantación comercial de algarrobo blanco (Prosopis alba) de 5 años de edad, en la localidad de Herrera (Dpto. Avellaneda) de la provincia de Santiago del Estero, Argentina, mediante un estudio de tiempos y movimientos para conocer la productividad operacional y los costos asociados con el proceso de apeo con motosierra. Se midieron tiempos efectivos y operativos y se calculó volumen con corteza, eficiencia, productividad y costo directo del raleo. La muestra de 226 árboles completó un volumen abatido de 1,19 $\mathrm{m}^{3}$. El tiempo operativo promedio fue 77 s/árbol y el tiempo efectivo medio de 49 s/árbol. Se verificó una fuerte correlación entre el tiempo efectivo de trabajo de la herramienta y las variables diámetro mayor y volumen de los árboles. La eficiencia operacional fue del 63,3\%. La productividad horaria en el derribo fue de 0,25 $\mathrm{m}^{3} / \mathrm{h}(47$ árboles/h). El costo diario de la operación fue de 781 \$/día, mientras que el costo unitario del raleo fue de 527 \$m3 (1 us $\$=8,12$ \$ en mayo 2014).

Palabras clave: raleo, costos, tiempo operativo, productividad.

Estimate of The Cost of The First ThinNing Methodology for Algarrobo blanco (Prosopis Alba): Applied in the Case of a Plantation in Herrera, Santiago Del Estero, Argentina

Abstract. The first forest thinning operation at a five-year-old algarrobo blanco (Prosopis alba) commercial plantation in Herrera (Department of Avellaneda) in the province of Santiago del Estero, Argentina was analyzed using a time and movement study to determine operational productivity and costs associated with the shoring process using a chainsaw. Effective and operative times were measured and the volume was calculated with respect to bark, efficiency, productivity and the direct cost of thinning. The sample of 226 trees had a cut volume of $1.19 \mathrm{~m}^{3}$. The average operative time was $77 \mathrm{~s} /$ tree and the median effective time was $49 \mathrm{~s} /$ tree. A strong correlation was found between the effective working time with the tool and the variables for greater diameter and volume of the trees. The operational efficiency was $63.3 \%$. Productivity per hour when felling trees was $0.25 \mathrm{~m}^{3} / \mathrm{h}(47$ trees $/ \mathrm{h})$. The daily cost of the operation was $781 \$$ /day, and the unit thinning cost was $527 \$ / \mathrm{m} 3$ ( 1 US\$ $=8.12 \$$ in May of 2014).

Keywords: thinning, costs, operative time, productivity.

\section{ESTIMATIVA DO CUSTO DO PRIMEIRO DESBASTE de Prosopis alba: Metodologia aplicada ao Caso de uma plantaÇão em Herrera, Santiago del Estero, Argentina}

Resumo. Analisou-se a operação do primeiro desbaste florestal numa plantação comercial de Prosopis alba de cinco anos de idade, em Herrera (Avellaneda) da província de Santiago del Estero (Argentina), mediante um estudo de tempos e movimentos para conhecer a produtividade operacional e os custos associados ao processo de abate com motosserra. Mediram-se tempos efetivos e operativos e calcularam-se volume com corte, eficiência, produtividade e custo direto do desbaste. A amostra de 226 árvores completou um volume abatido de $1,19 \mathrm{~m}^{3}$. O tempo operativo médio foi de 77s/árvore e o tempo efetivo médio de 49 s/árvore. Verificou-se uma forte correlação entre o tempo efetivo de trabalho da ferramenta e as variáveis diâmetro maior e volume das árvores. A eficiência operacional foi de $63,3 \%$. A produtividade horária na derrubada foi de $0,25 \mathrm{~m}^{3} / \mathrm{h}$ (47 árvores/h). O custo diário da operação foi de $781 \$$ por dia, enquanto o custo unitário do desbaste foi de $527 \$ / \mathrm{m}^{3}$ (1 US\$ = 8,12 \$ em maio 2014).

Palavras-chave: desbaste, custos, tempo operativo, produtividade. 


\section{Introducción 1}

El algarrobo blanco (Prosopis alba) es una especie de amplia distribución geográfica que crece en forma natural al norte de Argentina, en el Parque Chaqueño, región a la que pertenece casi toda la provincia de Santiago del Estero. Es un árbol de mediano porte de hasta $12 \mathrm{~m}$ de altura y un tronco de hasta $150 \mathrm{~cm}$ de diámetro [1].

En toda la región del Parque Chaqueño, y en particular Santiago del Estero, el algarrobo blanco es la principal especie nativa promocionada para realizar plantaciones comerciales, en el marco de la Ley Nacional 25.080/99 - y su reforma, la Ley 26.432/08 - que incentiva la forestación de especies nativas y exóticas en todo el territorio nacional, mediante planes de subsidio.

Las investigaciones sobre aspectos técnicos del manejo de plantaciones de algarrobo blanco en el Parque Chaqueño cobran mayor impulso desde la sanción de la mencionada Ley. Ewens y Navall [2] investigan sobre densidad de plantación y tratamiento de poda en Santiago del Estero; Delvalle [3] ensaya la intensidad del raleo en Chaco, y Pérez [4] describe la técnica de raleo para pequeños forestadores de Formosa; Kees, Gómez, Verga y Muttoni [5] obtienen funciones de volumen para forestaciones en Chaco.

Sin embargo, son escasos los antecedentes sobre los aspectos económicos del algarrobo blanco tales como rendimientos y costos de las diferentes etapas de la vida de la plantación. En Formosa, Vega [6] calcula rendimientos y costos de poda. En Santiago del Estero, los trabajos de Coronel de Renolfi, Cardona y Ruiz [7] y Coronel de Renolfi, Cardona, Ewens e Ibarra [8] estiman los costos de implantación y de vivero, respectivamente. No obstante, existe un vacío de información en lo que respecta al análisis económico del tratamiento silvícola de raleo.

El raleo es una práctica forestal mediante la cual se eliminan individuos indeseables, dejando en pie y bien distribuidos en el espacio, los mejores árboles de una plantación. Durante la vida del algarrobal se deben ejecutar 3 o 4 raleos hasta que llegue el momento de la corta final. El primer raleo ("raleo por lo bajo") es una corta de saneamiento que se realiza por única

1 Artículo de investigación generado en el proyecto de investigación "Evaluación de la calidad de madera para usos sólidos en especies maderables actuales y potenciales de la región Chaqueña" (2012-2015), de la Universidad Nacional de Santiago del Estero, Argentina. Proyecto financiado por el CICyT-UNSE, 2012-2015. vez, a temprana edad, cuando la plantación presenta un promedio menor a $10 \mathrm{~cm}$ de diámetro; tiene como fin depurar y mejorar la estructura de la masa forestal. Los siguientes raleos ("raleos comerciales") se ponen en práctica en distintos momentos de la plantación; se efectúan cuando las ramas de la copa de los árboles se tocan o están próximas a tocarse. El primer raleo comercial debe practicarse cuando el algarrobal tiene un promedio mayor a $10 \mathrm{~cm}$ de diámetro. En los raleos comerciales, los productos obtenidos son de dimensiones y calidades suficientes que permiten su industrialización.

El raleo forestal es una actividad de alto costo, pero imprescindible en la mayoría de las especies; tiene un gran impacto en la valoración del producto final. Existe la tendencia lógica de postergar los raleos hasta que haya un producto comercial para compensar el costo de este. Esta postergación del raleo es peligrosa y no recomendable. Particularmente, el primer raleo tiene más costos que beneficios; si se lo retrasa con el propósito de que sea comercial, se corre el riesgo de no lograr una plantación de calidad [9].

El cálculo del costo del raleo impone medir previamente tiempos y productividad de la labor silvícola, información que se obtiene con un estudio del trabajo. Según Tolosana [10], Villagómez [11] y Nájera Luna, Aguirre, Treviño, Jiménez y Jurado [12], el objetivo de un estudio del trabajo es la estimación de la productividad y los costos en la organización de la tarea.

El raleo puede ser selectivo o sistemático. En raleos selectivos, se inspecciona cada árbol para decidir cuáles individuos se van a eliminar y cuáles quedan en pie. En raleos sistemáticos, en cambio, se sigue un esquema preestablecido, en el cual no se considera la calidad de los árboles [7].

En este contexto, y con el fin de generar información económica del tema, el objetivo del presente estudio es determinar la productividad con el fin de estimar los costos de la primera intervención de raleo en una plantación de algarrobo blanco en Santiago del Estero.

\section{Materiales y métodos}

\section{1 Área de estudio}

El estudio de la operación de raleo se llevó a cabo en una plantación comercial de algarrobo blanco ubicada en la localidad de Herrera (Dpto. Avellaneda, provincia de Santiago del Estero), a $170 \mathrm{~km}$ de distancia al sudeste de la ciudad de Santiago del Estero (28 $28^{\circ}$ 'S y 
$\left.63^{\circ} 07^{\prime} \mathrm{W}\right)$. El algarrobal, con una superficie de 30 ha $y$ espaciamiento $8 \times 2 \mathrm{~m}$, fue implantado en el 2008 para su uso en un sistema silvopastoril.

\subsection{Toma de datos y mediciones}

Se registraron los tiempos de trabajo efectivos y operativos; se calculó volumen, eficiencia, productividad y costo del primer raleo (raleo por lo bajo).

Esta primera intervención de raleo se realizó en junio de 2013, a los 5 años de edad del algarrobal. Se aplicó un raleo sistemático a lo largo de 6 líneas de plantación, siguiendo la regla de selección de los ejemplares de "uno si-uno no" en cada línea (intensidad de raleo del 50\%). El sitio exhibía condiciones de limpieza $\mathrm{y}$ alta transitabilidad. El total de observaciones conformó una muestra de 226 individuos.

La operación fue ejecutada por dos operarios (motosierrista y ayudante), con nivel medio de experiencia en operaciones de apeo. El operador de la máquina utilizó una motosierra marca STIHL. El método de cosecha consistió en las operaciones de corte, volteo y desrame, sin incluir trozado del tronco, ordenamiento ni reducción de las ramas. La tarea del motosierrista fue la de apear el árbol y desramar la copa; el auxiliar colaboró manualmente en la dirección de caída, el volteo y el desrame. El tronco y las ramas separadas quedaron en el suelo.

La toma de datos se realizó con la técnica de observación directa, midiendo in situ los tiempos de trabajo [13] y registrándolos en planillas predefinidas. Los tiempos se midieron con cronómetro de precisión utilizando el método de vuelta a cero [14] en cada observación. Cada fase comprendió los siguientes movimientos: traslado-ubicación del árbol-preparación, corte, volteo y desrame. Para cada individuo, se registraron por separado: a) tiempo de traslado, ubicación frente al árbol y preparación para el corte, b) tiempo de inicio y fin de la tarea de corte (desde que se pone en marcha la motosierra hasta que finaliza el desrame) y c) tiempo que insumen las interrupciones de la tarea. La siguiente observación comenzó al iniciarse el traslado al próximo árbol. La distancia media de avance fue de 4 $\mathrm{m}$. No se tomaron tiempos de extracción ni transporte del material leñoso debido a que, a la fecha de la toma de datos, aun no se tenía previsto el destino del raleo.

Luego del desrame, se midieron con cinta métrica las dimensiones del tronco abatido (longitud, diámetros mayor y menor con corteza) a fin de determinar su volumen. Los tiempos cronometrados fueron los siguientes [15]: tiempo efectivo total, tiempos improductivos y tiempo operativo total (en minutos centesimales). Se calculó la eficiencia operacional del raleo $(\% \mathrm{E})$, definida como la relación porcentual entre los tiempos efectivos y tiempos operativos totales [16]:

$$
\% E=\frac{\sum_{T E F}}{\sum T O P} * 100
$$

\section{Donde:}

TEF: tiempo efectivo total. Es la suma del tiempo de ubicación, tiempo de trabajo efectivo (TTE) y los tiempos de traslado, sin incluir interrupciones o atrasos. El tiempo de trabajo efectivo es la parte del TEF durante el cual la motosierra funciona realizando el corte, volteo y desrame.

TI: tiempos improductivos. Es la suma de los tiempos ocasionales, no productivos o interrupciones que se producen durante la jornada de trabajo (charlas técnicas, descansos breves, carga de combustible, afilado de cadena de la motosierra, entre otros).

TOP: tiempo operativo total. Resulta de la adición de los dos tiempos parciales anteriores. Es el tiempo total que insume efectuar el trabajo de raleo, incluyendo los tiempos no productivos.

El volumen raleado se cubicó con la fórmula de Smalian [17], como se expresa en la ecuación 2.

$$
V_{T}=\frac{\pi}{4} *\left(\frac{D+d}{2}\right)^{2} * L
$$

\section{Donde: $V_{T}$ : volumen del árbol $\left(\mathrm{m}^{3}\right.$ con corteza) $D$ : diámetro mayor a la altura del corte ( $\mathrm{m} \mathrm{cc}$ ) $L$ : longitud del fuste $(\mathrm{m})$ \\ 2.3 Estimación de la productividad y los costos} $d$ : diámetro menor a la altura del despunte $(\mathrm{m} \mathrm{cc})$

Según Nájera Luna [18], la productividad relaciona la cantidad de trabajo efectuado por unidad de tiempo. En el presente trabajo, la productividad horaria de la tarea de raleo $(\mathrm{P})$ se calculó dividiendo el resultado obtenido (cantidad de árboles raleados y volumen c/c apeado) por el Top, tiempo operativo total expresado en horas [19].

Se estimó la productividad diaria (PD), proyectando el número de árboles y la cantidad $\mathrm{de}^{3}$ que 
se pueden ralear por jornada de trabajo; a esta última se asignó una base de 6 horas activas de trabajo (jornada laboral de $8 \mathrm{~h}$, descontadas $2 \mathrm{~h}$ de descansos reglamentarios).

El costo unitario del raleo se determinó según la Organización de las Naciones Unidas para la Alimentación y la Agricultura (FAO, en sus siglas en inglés) [20] y representa lo que cuesta realizar la tarea por unidad de producción [21]; se trata de un costo directo que incluye los costos operativos de la motosierra y de la mano de obra, conforme a la ecuación 3.

$$
C R=\frac{[s(1+\operatorname{CsS} c)+C M]}{P D}
$$

Donde:

CR: $\quad$ costo unitario del raleo $\left(\mathrm{en} \$ / \mathrm{m}^{3}\right.$ )

$s: \quad$ salarios del jornal; motosierrista y ayudante (\$/día)

CSSC: cargas sociales (60\% del salario)

CM: $\quad$ costo operativo de la motosierra (\$/día)

$P D$ : productividad diaria ( $\mathrm{m}^{3} /$ día)

Los salarios s correspondieron a las escalas salariales vigentes a diciembre de 2013, para personal permanente (categorías motosierrista y peón general) comprendido en el Régimen de Trabajo Agrario de Argentina [22].

Para el cálculo del costo operativo de la motosierra (См), se utilizaron los precios vigentes a mayo de 2014, aplicando la metodología propuesta por Frank [23] y Coronel de Renolfi y Cardona [24], según la ecuación 4: $C M=(i+a+c c+c l+r y m) *$ TTE, donde $i$ es interés de la máquina $(\$ / \mathrm{h}), a$ es el costo por amortización $(\$ / \mathrm{h}), c c$ es el gasto de consumo de combustible mezcla $(\$ / \mathrm{h}), \mathrm{cl}$ es el gasto de consumo de lubricante de cadena $(\$ / \mathrm{h})$, rym es el gasto de reparación y mantenimiento $(\$ / \mathrm{h})$ y TTE es el tiempo de trabajo efectivo con la motosierra (h/día).

Se utilizó una motosierra nueva, marca STIHL Ms 250, cilindro de 45,4 cc, depósito de combustible de 0,47 litros, espada de 45/1,3R, cadena 3/8" 36 RM y peso neto de $4,6 \mathrm{~kg}$. El motor de dos tiempos requiere de combustible mezcla de nafta y aceite en proporción 1:50. La tabla 1 resume los datos técnicos y económicos de base para el cálculo del costo operativo.

Tabla 1. Información técnico-económica para el cálculo del costo de la motosierra

\begin{tabular}{|l|l|c|}
\hline \multicolumn{2}{|c|}{ Ítems } & Valor \\
\hline Motosierra & Valor a nuevo (\$) & 4416 \\
\hline & Vida útil (h) & 2000 \\
\hline & Potencia (HP) & 3,10 \\
\hline & Interés (\% anual) & 8,00 \\
\hline & Precio combustible mezcla $(\$ /$ litro $)$ & 15,54 \\
\hline & Consumo estimado combustible $(1 / \mathrm{h})$ & 0,94 \\
\hline & Precio lubricante cadena $(\$ / 1)$ & 53,00 \\
\hline & Consumo estimado de lubricante $(1 / \mathrm{h})$ & 0,33 \\
\hline & Reparación y mantenimiento $(\%$ de a) & 40,00 \\
\hline & Jornal motosierrista c/Cs Sc $(\$ /$ día) & 348,66 \\
\hline & Jornal peón auxiliar c/Cs Sc $(\$ /$ día) & 312,54 \\
\hline
\end{tabular}

Fuente: elaboración propia con datos del mercado local 1 US $\$=8,12 \$$ (28 de mayo de 2014)

\section{Resultados y discusión}

\subsection{Volumen, tiempos de trabajo y productividad del raleo}

En la tabla 2, se presentan los estadísticos descriptivos de las variables largo, diámetros mayor y menor y volumen con corteza de los árboles apeados. El volumen unitario del apeo (volumen medio por árbol) fue de $0,00526 \mathrm{~m}^{3}$ cc, de modo que el raleo de los 226 árboles de la muestra completó un volumen total de $1,18891 \mathrm{~m}^{3}$.

Según la tabla 2, los individuos medidos presentaron dimensiones bastante similares, con una variabilidad entre el 30-34\%, mientras que el volumen del raleo presentó gran variabilidad $(72,68 \%)$. La variable diámetro mayor (ordenada por clases) mostró una distribución normal de frecuencias (figura 1). La distribu-

Tabla 2. Estadísticos descriptivos de las variables largo, diámetros y volumen del raleo

\begin{tabular}{|l|c|c|c|c|c|}
\hline \multicolumn{1}{|c|}{ Variable } & Media & Desvío Est. & Cv (\%) & Max & Min \\
\hline Diámetro mayor $(\mathrm{cm})$ & 7,72 & 2,37 & 30,68 & 14,01 & 1,59 \\
\hline Diámetro menor $(\mathrm{cm})$ & 5,59 & 1,9 & 33,99 & 10,98 & 1,58 \\
\hline Longitud $(\mathrm{m})$ & 1,27 & 0,38 & 30,07 & 2,25 & 0,2 \\
\hline Volumen $\left(\mathrm{m}^{3} \mathrm{cc}\right)$ & 0,00526 & 0,00382 & 72,68 & 0,02193 & 0,00004 \\
\hline
\end{tabular}

Fuente: elaboración propia 


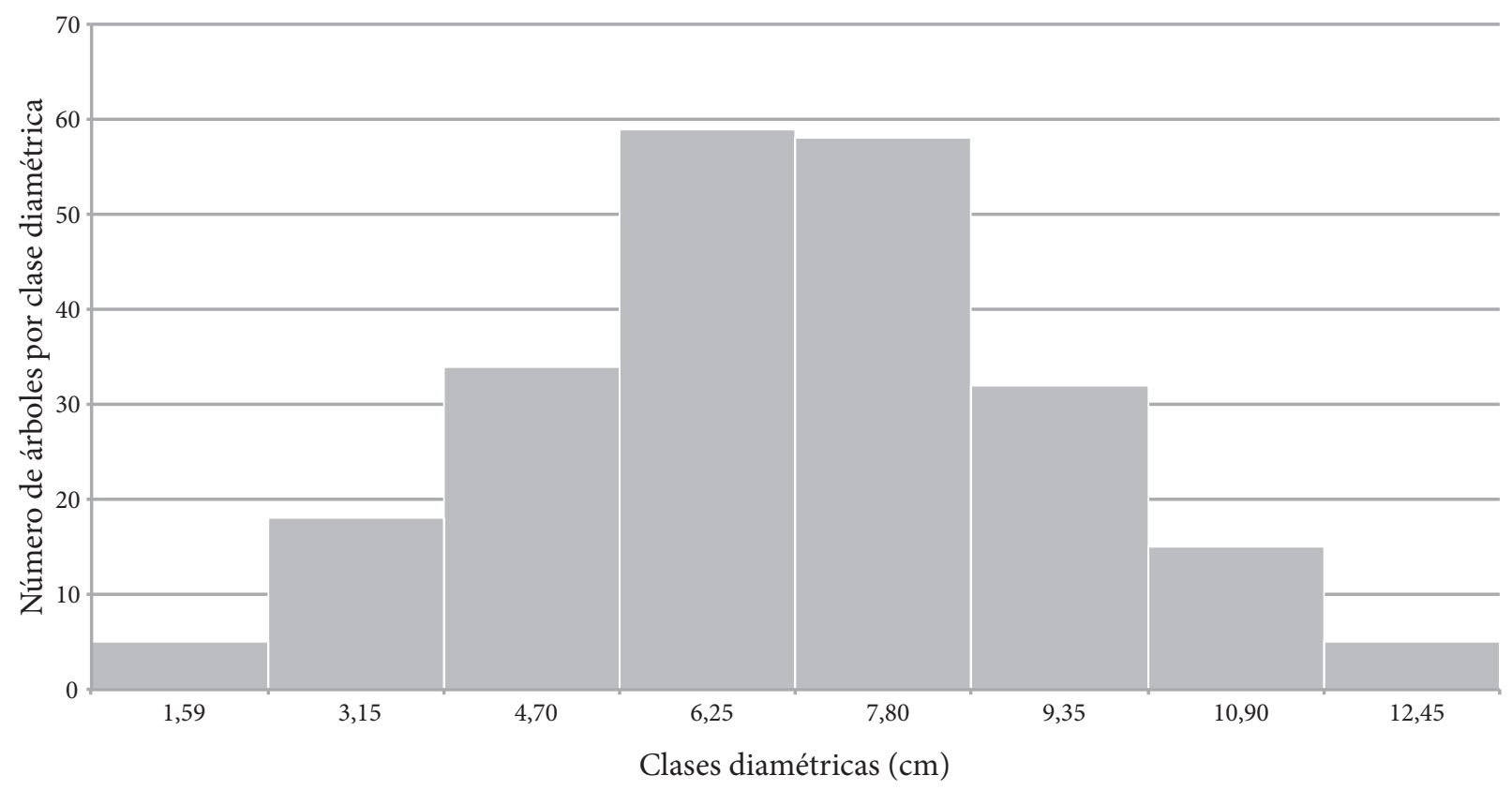

Figura 1. Distribución de frecuencias del número de árboles apeados por clases de diámetro Fuente: elaboración propia

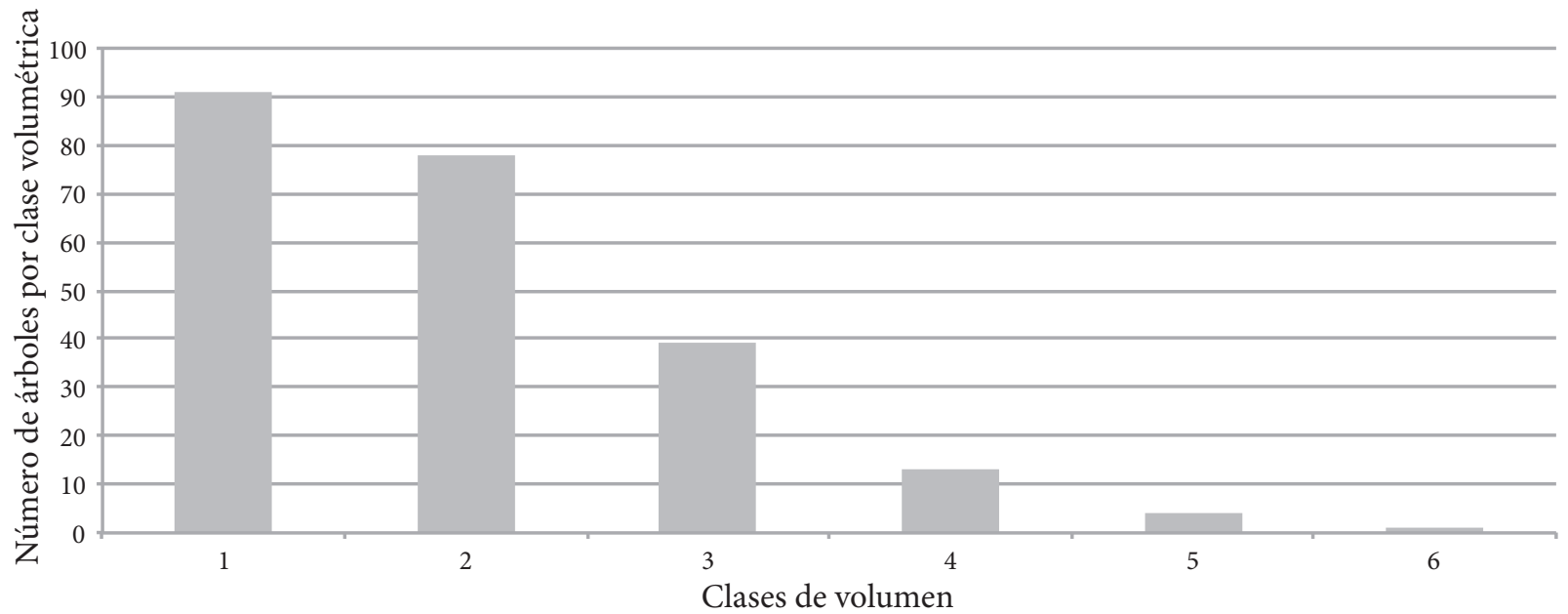

Figura 2. Distribución de frecuencias del número de árboles raleados por clases de volumen Fuente: elaboración propia

ción de frecuencias agrupadas para la variable volumen se exhibe la figura 2, donde se observa que el $92 \%$ de los individuos no supera un volumen individual de 0,010 $\mathrm{m}^{3}$ (clase 3 de volumen).

Dado que las filas de plantación no tenían la misma cantidad de individuos, el número de árboles ralea- dos en cada fila fue diferente. No obstante, el aporte de cada una de las 6 filas al volumen total fue bastante uniforme, excepto la fila 5 (ver figura 3). El volumen medio por fila se muestra en la figura 4 . 


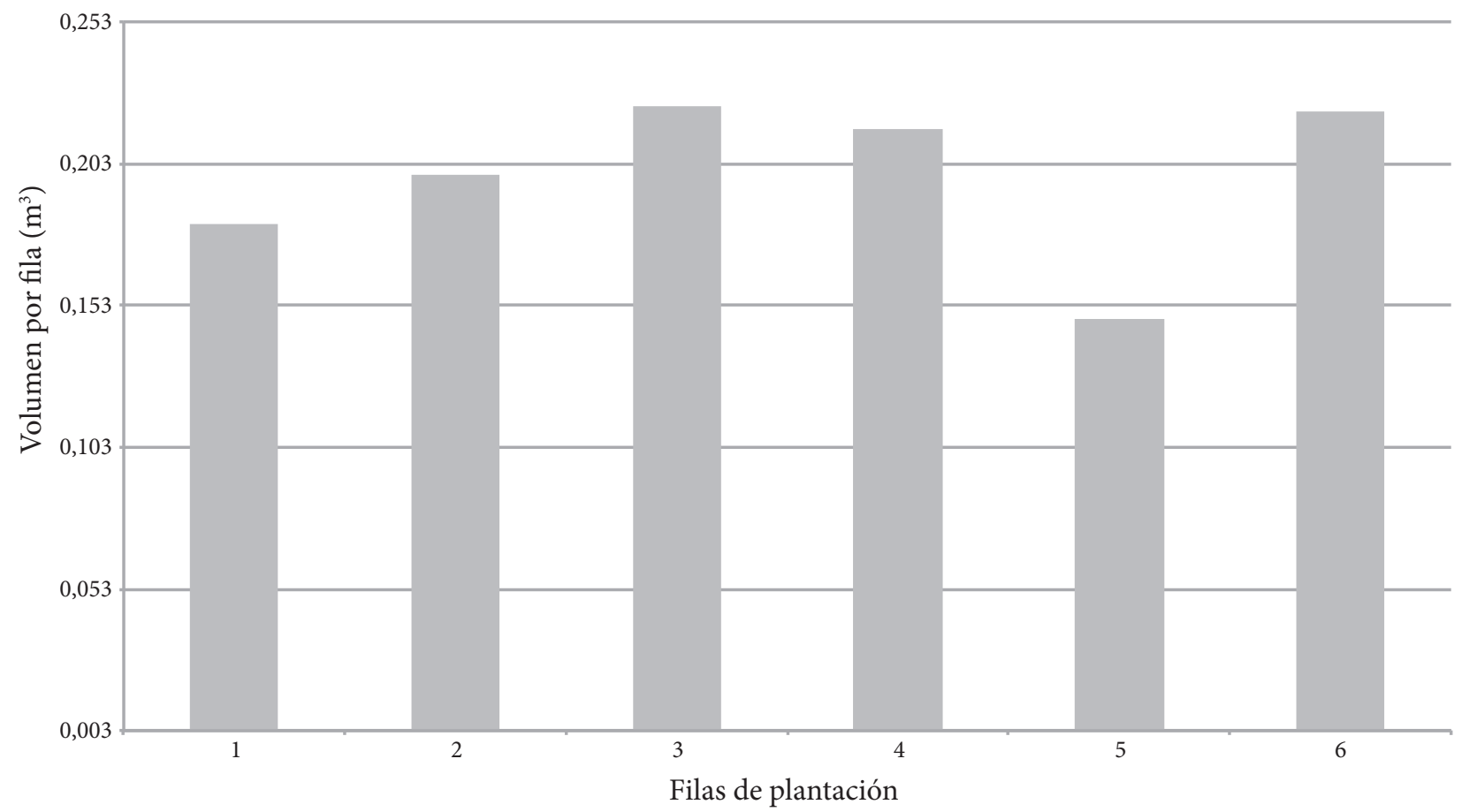

Figura 3. Volumen total raleado por fila de plantación

Fuente: elaboración propia

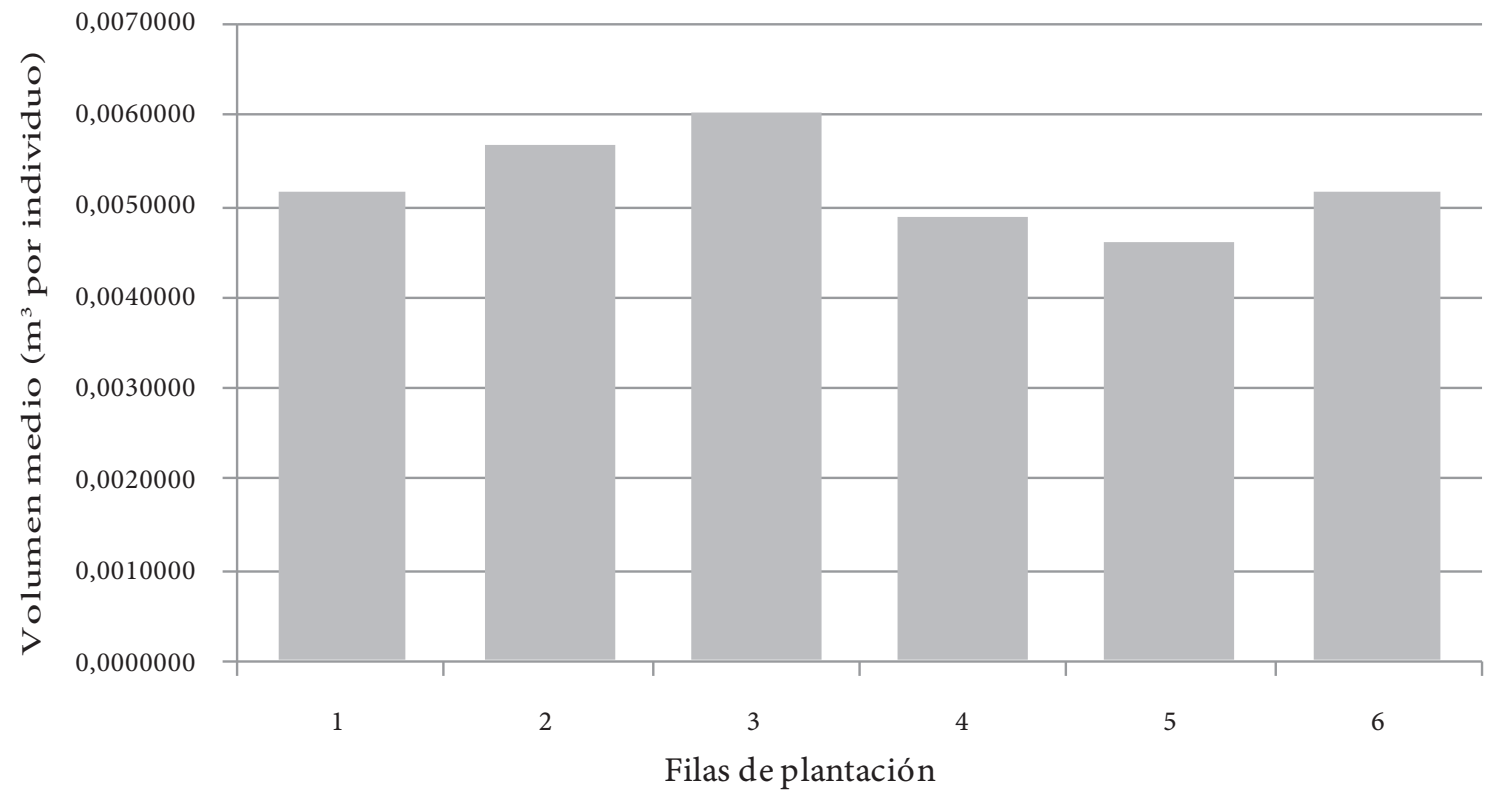

Figura 4. Volumen promedio del raleo por fila de plantación

Fuente: elaboración propia

La tabla 3 expone los resultados de la toma de tiempos correspondientes a los promedios del tiempo efectivo total (TEF), tiempos improductivos (TI) y tiempo operativo total (TOP), expresados en segundos por árbol y en minutos por metro cúbico. Los porcentajes de contribución en el TOP fueron de un 63,3\% el TEF y un 36,7\% los TI. A su vez, el TEF se desglosa en el tiempo de trabajo efectivo de la motosierra (TTE) y el tiem- 
po de traslado con el $53,0 \%$ y el $10,3 \%$ de participación, respectivamente. El 63,3\% de contribución del TEF en el TOP representa, según la ecuación 1, la eficiencia operacional del raleo.

Tabla 3. Promedios de los tiempos de trabajo del raleo

\begin{tabular}{|l|c|c|}
\hline \multicolumn{1}{|c|}{ Tiempos promedios } & Segundos/árbol & Minutos $/ \mathbf{m}^{\mathbf{3}}$ \\
\hline Tiempo efectivo total & 48,56 & 153,84 \\
\hline Tiempos improductivos & 28,15 & 89,20 \\
\hline Tiempo operativo total & 76,71 & 243,04 \\
\hline
\end{tabular}

Fuente: elaboración propia

Los tiempos improductivos correspondieron a los momentos de carga de combustible, afilado de la cadena y pequeños descansos, cuya distribución porcentual se presenta en la figura 5.

Nájera Luna, Aguirre, Treviño, Jiménez y Jurado [12] señalan que el tamaño de los árboles es un factor que afecta el rendimiento del derribo manual con motosierra. Para evaluar la relación existente entre tiempos de trabajo y dimensión de los árboles, se realizó un análisis de regresión utilizando rutinas del programa Excel 2007 ๑. Se probaron como variables independientes, el diámetro mayor y el volumen, ordenados por clases o categorías; y como variables dependiente, el TOP y el TTE.
No se halló una ecuación de buen ajuste entre volumen у TOP, ni entre diámetro y TOP. Por el contrario, la ecuación que relaciona el volumen apeado y el TTE (figura 6) reveló una buena correlación entre estas dos variables, con un coeficiente de determinación $R^{2}=0,764$. Asimismo se halló una relación lineal directa entre diámetro mayor y TTE en la que el valor del coeficiente $R^{2}$ indicó un buen ajuste de la siguiente función: $Y=2,246 x+$ $25,909, R^{2}=0,7093$, donde $Y$ es TTE (en segundos centesimales) y $x$ es categorías de diámetro mayor.

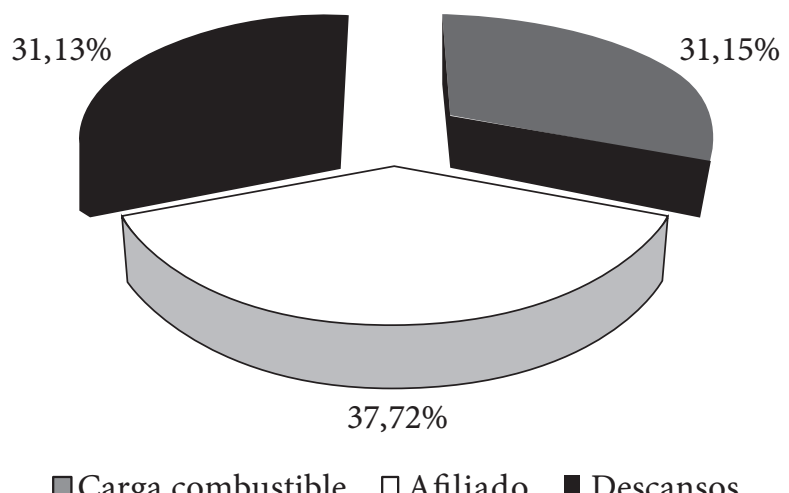

Figura 5. Distribución porcentual de los diferentes ítems del tiempo improductivo

Fuente: elaboración propia

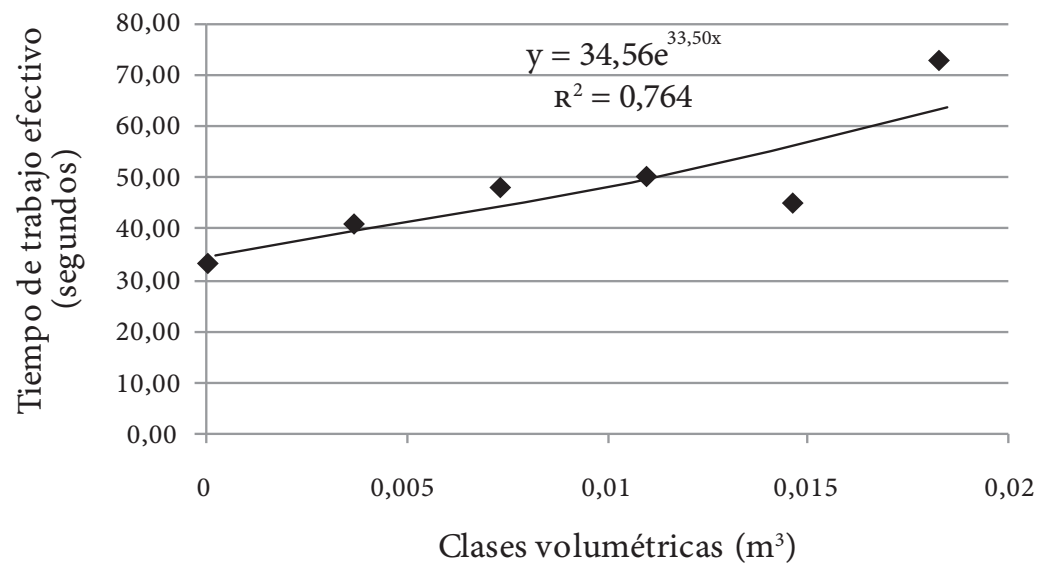

Figura 6. Función de ajuste entre tiempo de trabajo efectivo y volumen del raleo. $Y=$ TTE (en segundos centesimales); $x=$ clases de volumen

Fuente: elaboración propia 
Se estimó la productividad horaria media del raleo (en $\mathrm{N}^{\circ}{ }^{\circ}$ árboles/hora y $\mathrm{m}^{3}$ /hora) y la productividad diaria (PD). La tabla 4 resume los valores hallados de eficiencia y productividad de la tarea.

Tabla 4. Eficiencia y productividad media del raleo

\begin{tabular}{|l|c|}
\hline \multicolumn{1}{|c|}{ Variable } & Valor \\
\hline Eficiencia (\%) & 63,30 \\
\hline Productividad horaria (árboles/h) & 46,93 \\
\hline Productividad horaria $\left(\mathrm{m}^{3} / \mathrm{h}\right)$ & 0,25 \\
\hline Productividad diaria (árboles/día & 281,57 \\
\hline Productividad diaria (m³/día) & 1,48 \\
\hline
\end{tabular}

Fuente: elaboración propia

Cabe advertir que la falta de estudios locales sobre tiempos de trabajo, eficiencia y productividad en operaciones de raleo impide tener referencias para efectuar comparaciones válidas.

\subsection{Costo del raleo}

Con los datos técnicos y económicos de la tabla 1 y usando la ecuación 4 , se calculó el costo operativo horario y diario de la motosierra (ver tabla 5). El tiempo de trabajo efectivo TTE de la máquina, expresado en horas por día, se estimó sobre la base de 6 horas de trabajo activo, aplicando idéntico criterio que para proyectar la productividad diaria. Por tanto, si el TTE representa el 53\% del tiempo total TOP, el uso diario resultó en 3,18 horas/día.

Tabla 5. Costo operativo de la motosierra

\begin{tabular}{|l|c|}
\hline \multicolumn{1}{|c|}{ Ítems del costo } & Valor \\
\hline Interés (i) & $0,18 \$ / \mathrm{h}$ \\
\hline Amortización (a) & $3,90 \$ / \mathrm{h}$ \\
\hline Gasto consumo combustible mezcla (cc) & $14,61 \$ / \mathrm{h}$ \\
\hline Gasto consumo lubricante cadena (cl) & $17,44 \$ / \mathrm{h}$ \\
\hline Gasto reparación y mantenimiento (rym) & $1,56 \$ / \mathrm{h}$ \\
\hline Costo operativo horario $=$ & $14,61 \$ / \mathrm{h}$ \\
\hline Tiempo trabajo efectivo (TTE) & $3,18 \mathrm{~h} /$ día \\
\hline Costo operativo diario $(\mathrm{CM})=$ & $119,83 \$ /$ día \\
\hline
\end{tabular}

Fuente: elaboración propia

1 US\$ $=8,12 \$$ (28 de mayo 2014)

Según la tabla 5, en el costo horario dela herramienta se destaca la incidencia del gasto de lubricación de la cadena, con un $46,3 \%$. El costo diario de la tarea (mano de obra directa y motosierra) ascendió a 781 \$/día, en el que la carga de la mano de obra representa el $85 \%$ de dicho total.

Con base en el valor de productividad diaria obtenida en este caso de estudio (tabla 4) y aplicando la ecuación 3, resultó un costo unitario del raleo CR de $527,28 \$ / \mathrm{m}^{3}$.

\section{Conclusiones}

El tiempo operativo promedio del raleo es de 1,28 minutos por árbol, del cual el 63,3\% pertenece al tiempo efectivo. Durante los tiempos no productivos, las detenciones por afilado de la cadena representan el casi $38 \%$ de aquellos.

El volumen promedio del raleo es de $0,00526 \mathrm{~m}^{3} \mathrm{cc}$ por árbol y presenta gran variabilidad. Se verifica la existencia de una correlación de buen ajuste estadístico entre el tiempo de trabajo efectivo de la motosierra y el volumen del raleo, como así también entre dicho tiempo y el diámetro a la altura del corte. En el primer caso se identifica una relación exponencial y el segundo, una relación lineal directa entre las variables.

La productividad de la operación es de 282 árboles/día (1,48 m³/día). El costo diario de la operación es de $781 \$$, mientras que el costo unitario del raleo es de $527,28 \$ / \mathrm{m}^{3}$, valor equivalente a $2,77 \$$ /árbol.

Si bien el costo operativo calculado para la motosierra es un valor independiente, cabe advertir que los demás valores obtenidos (de eficiencia, productividad y costo del raleo) sólo son aplicables en el escenario particular del caso de estudio porque están en función de las dimensiones de los árboles, de la destreza de los operarios y de la organización de la tarea.

La metodología aplicada para calcular productividad y costo del raleo resulta adecuada; los resultados constituyen antecedentes iniciales en la zona y sientan las bases para futuros cálculos.

\section{Referencias}

[1] L.A. Tortorelli, Maderas y bosques argentinos, Tomo 1, $2^{a}$ ed. Buenos Aires: Orientación Gráfica Editora, 2009, $515 \mathrm{p}$.

[2] M. Ewens y M. Navall, "Silvicultura del algarrobo blanco", $2^{\circ}$ Jornadas Forestales de Santiago del Estero, Junio, Santiago del Estero, 2006, 7p.

[3] P. Delvalle, "Raleo selectivo en forestación joven de algarrobo blanco", $2^{\circ}$ Jornadas Forestales de Santiago del Estero, Junio, Santiago del Estero, 2006, 6p. 
[4] V. Pérez, "Raleo en forestación de algarrobos", 23 de abril 2014. Disponible en http://chaco-forestal.blogspot.com.ar/p/informacion-tecnica_20.html

[5] S. M. Kees, C. Gómez, A. Verga y F. Muttoni, "Funciones de volumen de fuste para árboles individuales de forestaciones con Prosopis alba Griseb (algarrobo blanco) en la provincia de Chaco", Reunión Nacional del Algarrobo. Noviembre, Córdoba, pp. 64-65, 2012.

[6] C. Vega, Rendimientos operativos y costos de poda en Prosopis alba Griseb. Tesis Ingeniería Forestal. Formosa: Universidad Nacional de Formosa, 2009, 79p.

[7] M. Coronel de Renolfi, G. Cardona y A. Ruiz, "Coeficientes técnicos del primer año de plantación de Prosopis sp. en Santiago del Estero", Quebracho, vol. 18, pp. 58-70, 2010.

[8] M. Coronel de Renolfi, G. Cardona, M. Ewens y E. Ibarra, "Productividad y requerimientos de mano de obra en la producción comercial de plantines de algarrobo blanco (Prosopis alba). El caso del vivero forestal de la Estación Experimental Fernández, Santiago del Estero", Quebracho, vol. 21, pp. 26-38, 2013.

[9] G. Galloway, "Manejo de plantaciones forestales: guía técnica para el extensionista", Centro Agronómico Tropical de Investigación y Enseñanza, Serie técnica, vol. 7, pp. 27-44, 1993.

[10] E. Tolosana, "El aprovechamiento forestal mecanizado en las cortas de mejora de Pinus sylvestris L. Modelos de tiempos, rendimientos y costes y estudio de sus efectos ambientales", Tesis doctoral. Madrid: Universidad Politécnica de Madrid. ETSI Montes, 1999, 222p.

[11] M. A. Villagómez, "Selección de maquinaria para la operación de derribo en bosques de coníferas". Revista Mexicana de Ciencias Forestales, vol.2, n. ${ }^{\circ}$, pp. 91-103, 2011, pp. 91-103.

[12] J. A. Nájera Luna, O. Aguirre, E. Treviño, J. Jiménez y E. Jurado, "Tiempos y rendimientos del aprovechamiento forestal en El Salto, Durango, México", Revista Chapingo, Serie Ciencias Forestales, vol. 17, n. ${ }^{\circ} 1$, pp. 58-69, 2011, pp. 58-69.

[13] B. W. Niebel y A. Freivalds, Ingeniería Industrial, Métodos, estándares y diseño del trabajo, vol. 1,10a ed. México D. F.: Alfaomega Grupo Editor, 2001, pp. 546-547.
[14] A. Caso Neira, Técnicas de medición del trabajo, 2a ed. Madrid: FC Editorial, 2006, 232p.

[15] P. Carey, A. Figueroa Sotomayor, P. Valenzuela Caviares, "Evaluación técnica de un sistema tradicional de cosecha en plantaciones de Eucalyptus globulus de corta rotación en Valdivia, Chile”, Bosques, vol. 27, n. ${ }^{\circ} 3,2006$, pp. 272-276.

[16] D. Simões, P. Torres Fenner y C. Bantel, "Análise Operacional e Económica do Processamento de Madeira de Eucalipto com Hypro em Regiao Montanhosa", Árvore, vol. 35, n. ${ }^{\circ}$ 3, pp. 505-514, 2011.

[17] B. Husch, C. Miller y T. Beers, Forest mensuration, $4^{\text {a }}$ ed. New Jersey: John Wiley \& Sons.Hoboken, 2003, 443p.

[18] J. A. Nájera Luna, "Evaluación del proceso productivo maderable en la región de El Salto, Durango, México", Tesis doctoral. Nueva León: Universidad Autónoma de Nueva León, Facultad de Ciencias Forestales, 2010, 198p.

[19] A. P. de Souza, L. Minette, F. Moreira, C. Machado y K. Silva, "Análise do desempenho da máquina slingshot em subsistemas de colheita em florestas de eucalipto", Revista Brasileira de Engenharia Agrícola e Ambiental, vol. 8, pp. 316-320, 2004.

[20] Organización de las Naciones Unidas para la Alimentación y la Agricultura, FAO, Evaluación de los costos de extracción a partir de inventarios forestales en los trópi$\cos , 2^{\text {a }}$ parte. Roma: FAO, 1978, 76p.

[21] E. Tolosana, V. González y S. Vignote, El aprovechamiento maderero. Madrid: Editorial Mundi Prensa, 2000, 563p.

[22] CNTA. Comisión Nacional del Trabajo Agrario, Resolución 103/12. 20 mayo 2014. Disponible en http://www. cra.org.ar/files/content/5/5949/res_cnta_103_2012. pdf.

[23] R. Frank, Introducción al cálculo de costos agropecuarios, $6^{\mathrm{a}}$ ed. Buenos Aires: Editorial El Ateneo, 1995, 39p.

[24] M. Coronel de Renolfi y G. Cardona, Cómo calcular costos forestales. Santiago del Estero: Editorial Lucrecia, 2011, 152p. 